Résumés des conférences et travaux

\title{
Langue et littératures néo-latines
}

\section{Virginie Leroux}

\section{OpenEdition \\ Journals}

\section{Édition électronique}

URL : https://journals.openedition.org/ashp/3041

DOI : 10.4000/ashp.3041

ISSN : 1969-6310

\section{Éditeur}

Publications de l'École Pratique des Hautes Études

\section{Édition imprimée}

Date de publication : 1 septembre 2019

Pagination : 242-246

ISSN : 0766-0677

\section{Référence électronique}

Virginie Leroux, "Langue et littératures néo-latines », Annuaire de l'École pratique des hautes études (EPHE), Section des sciences historiques et philologiques [En ligne], 150 |2019, mis en ligne le 11 juin 2019, consulté le 06 juillet 2021. URL : http://journals.openedition.org/ashp/3041 ; DOI : https:// doi.org/10.4000/ashp.3041 


\title{
LANGUE ET LITTÉRATURES NÉO-LATINES
}

\author{
Directeur d'études : M ${ }^{\text {me }}$ Virginie Leroux
}

Programme de l'année 2017-2018 : I. La théorie des genres encomiastiques dans les Poetices libri septem de Jules-César Scaliger (1561). - II. Georg Sabinus Schüler, dit Sabinus, poète de Charles Quint.

Les conférences de l'année ont porté sur la poésie encomiastique, abordée sous un double aspect, théorique et pratique.

\section{La théorie des genres encomiastiques dans les Poetices libri septem de Jules-César Scaliger (1561)}

Les Poetices libri septem de Jules-César Scaliger (Lyon, A. Vincent, 1561) témoignent d'un renouveau de la théorie générique stimulé par la diffusion de la Poétique d'Aristote et la parution des premiers commentaires humanistes. Francesco Robortello fait ainsi suivre son commentaire de la Poétique d'Aristote et sa paraphrase de l'Épître aux Pisons de cinq traités consacrés à des genres ou procédés poétiques négligés par le Stagirite (satire, épigramme, comédie, traits piquants et élégie) ${ }^{1}$. La présence précieuse à la conférence de Monique Bouquet (université de Rennes), qui organisa, en 2016, un colloque consacré à Robortello, dont les actes paraîtront aux PUR, et de Sylvaine Poujade qui a préparé sous la direction de cette dernière un doctorat consacré au commentaire de Robortello (soutenu le 8 décembre 2018) a donné lieu à de riches discussions et nous a permis de préciser ce que Robortello appelle la «méthode» du Stagirite, qu'il définit en s'appuyant sur le commentaire de Jean Philophon aux Seconds analytiques ${ }^{2}$. Nous avons ainsi pu réfléchir aux liens entre logique et poétique dans la continuité des discussions menées lors d'une journée d'étude du projet Schol'art ${ }^{3}$. Conciliant une visée normative et l'ambition de décrire l'origine et l'évolution des genres antiques, Robortello associe érudition et réflexion théorique : outre l'application d'outils conceptuels exploités par Aristote à des genres absents de la Poétique, son apport à la théorie des genres consiste à affiner les critères de description stylistique qu'il regroupe sous le terme artificium.

Publié deux années avant les Poetices libri septem, le De poeta d'Antonio Sebastiano Minturno, paru à Venise, chez F. Rampazetto, en 1559, distingue une poétique

1. Francesco Robortello, Paraphrasis in librum Horatii qui uulgo de arte poetica ad Pisones inscribitur. Eiusdem explicationes De satyra, De epigrammate, De comoedia, De salibus, De elegia quae omnia addita ab authore fuerunt, ut nihil quod ad poeticam spectaret desiderari posset: Nam in iis scribendis Aristotelis methodum seruauit : et ex ispius Libello de arte Poetica principia sumpsit omnium suarum explicationum, Florence, L. Torrentino, 1548.

2. Voir V. Leroux, «Robortello et la théorie des genres littéraires », colloque international Francesco Robortello, org. Monique Bouquet et Claire Lesage, Rennes II, 6-8 octobre 2016, à paraître dans les actes.

3. V. Leroux, « Logique et poétique : l'exemple de Robortello », Projet Schol'art. « Les theories modernes des lettres et des arts à la lumière de la scolastique (France-Italie, 1500-1700) 》, Journée de lancement, vendredi $1^{\text {er }}$ décembre 2017, Université catholique de Louvain. 
générale (livre I, début du livre II et livre VI) et une poétique consacrée aux genres poétiques (genre héroïque dans le livre II, tragédie dans le livre III, comédie dans le livre IV et poésie lyrique dans le livre V), division qui sera notamment adoptée par Giovanni Antonio Viperano et Iacobus Pontanus. Ses critères d'analyse générique sont, en partie, ceux qu'élabore Francesco Robortello, cependant il systématise une méthode d'analyse, en particulier lorsqu'il traite des genres lyriques. La première approche est historique et décrit l'origine du genre et son développement. Dans un second temps, des préceptes (praecepta) sont énoncés et l'exposé devient normatif. L'ordre suivi est sensiblement le même : après une caractérisation brève de l'officium du genre et une présentation générale selon les critères du style, de la matière ou du mode, est proposée une définition calquée sur la définition aristotélicienne de la tragédie. Sont ensuite décrites les parties (partes) du genre que les exégètes postérieurs appelleront «parties de qualité », héritées de la définition aristotélicienne de la tragédie (la fable, les caractères, les pensées et l'expression) et les " parties de quantité » qui concernent la structure et la disposition, tandis que l'exposé s'achève généralement par l'examen de l'artificium. Grâce aux interventions de Nathalie Dauvois (université Paris III) et de Roland Béhar (ENS, PSL), nous avons évalué la différence entre les analyses menées par Minturno dans son traité latin et dans le traité qu'il consacre à la poétique toscane, 1'Arte poetica, publié en 1564, par Giovanni Andrea Valvassori.

La structure des Poetices libri septem de Jules-César Scaliger entraîne une façon spécifique d'aborder les genres poétiques. Le livre I, Historicus, envisage selon un point de vue historique la naissance de la poésie et sa division en espèces. Les genres sont ainsi abordés suivant l'ordre chronologique : la poésie pastorale, examinée au chapitre 4, correspond au mode de vie le plus primitif. Suivent les genres dramatiques (comédie, tragédie, mime et drame satyrique) dont l'étude comprend l'histoire de la danse (I, 18), les modes et les flûtes (I, 19-20), les théâtres (I, 21) et les jeux grecs, puis latins (I, 22-39). La fin du livre est consacrée aux autres genres, successivement la rhapsodie et l'épopée (I, 41), la parodie (I, 42), les centons (I, 43), la poésie lyrique (I, 44), les hymnes (I, 45), les dithyrambes (I, 46), les $\sigma 0 ́ v \theta \varepsilon \tau \alpha$ et hyporchemata (I, 47), les harmonies et instruments lyriques (I, 48), les chœurs (I, 49), les thrènes, hyménées et élégies (I, 50), les poèmes ioniques (I, 51), les hilarodi (I, 52), les dirae (I, 53), les palinodies (I, 54), les chansons (I, 55) et divers genres d'épigrammes (I, 56-57). Il s'agit de rendre compte de toutes les catégories et de tous les noms de poèmes dans une perspective archéologique.

En revanche, au livre III, intitulé Idea, Scaliger fournit des préceptes (praecepta) et des lois (leges) génériques en suivant un ordre hiérarchique décroissant : l'examen s'ouvre par le genre épique (III, 95); suivent les genres dramatiques (III, 96), la satire (III, 97), la poésie pastorale (III, 98), les petits genres qui relèvent de la silve (III, 99-122), la poésie lyrique (III, 123), l'élégie (III, 124) et l'épigramme (III, 125, 126). François Lecercle a opposé deux formes distinctes de poétique des genres, une théorie énumérative et une théorie combinatoire à partir des critères aristotéliciens que sont l'instrument (mots, rythme, harmonie), la matière (meilleure, semblable, pire) et le mode (direct, indirect, mixte) ${ }^{4}$. Si Scaliger esquisse une théorie combinatoire au

4. «La compulsion taxinomique : Scaliger et la théorie des genres », dans La statue et l'empreinte, la poétique de Scaliger [actes du colloque du Centre d'études supérieures de la Renaissance de Tours, 1983], ét. réunies et présentées par C. Balavoine et P. Laurens, Paris, J. Vrin, 1986, p. 89-99. 
début du livre I de ses Poetices libri septem (I, 2, éd. 1561, p. 6A), dans le livre III, il se plaît aussi à énumérer de nombreux genres mineurs qui ne figurent pas dans les taxinomies antiques et qui relèvent de la poésie de circonstance, comme l'Épithalame (III, 100), le Généthliaque (III, 101), le Proseuktikon (III, 102), le Propemptikon et l'Apopemptikon (III, 103), le Protreptikon et la Parainetikon (III, 104), l'Épibaterion (III, 105), le Panégyrique (III, 108) et autres genres épidictiques. Nous avons traduit les chapitres 108 à 119. Le chapitre 108 est consacré au panégyrique qui a pour origine les célébrations solennelles des Grecs. Le chapitre 109 analyse les genres de

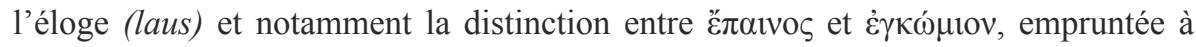
Alexandros, fils de Nouménios. Le chapitre 110 précise ce que chacun peut louer, du Seigneur à l'indolence du moineau. Le chapitre 111 est consacré aux hymnes et à leurs divisions, le 112 aux évocations et invocations, le 113 aux hymnes de congé

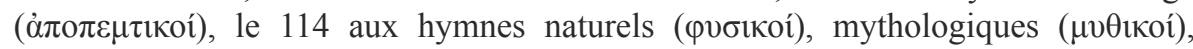

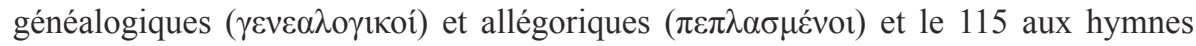
héroïques, Péans et Scolies. Le chapitre 116 définit les dithyrambes et le 117 les louanges (laudationes), examinées cette fois au prisme des matières louées, distinguées selon leur degré de dignité : arguments $\alpha \varkappa \delta o \xi \alpha$ (sans noblesse), ह̌ $v \delta o \xi \alpha$ (glorieux), $\not \alpha \mu \varphi 1 \delta o \xi \alpha$ (contestables) ou $\pi \alpha \rho \alpha ́ \delta o \xi \alpha$ (inattendus). Enfin, les chapitres 118 et 119 fournissent la topique de l'éloge d'une personne et d'un lieu. La traduction nous a permis de dégager quelques caractéristiques de la théorie des genres encomiastiques : en l'absence de modèle théorique poétique, Scaliger n'applique aucunement les critères aristotéliciens fournis par ses prédécesseurs, ni la théorie combinatoire qu'il exhibe dans son traité, mais il accorde une grande attention à l'étymologie, à l'origine et au contexte anthropologique dans lequel apparurent et furent utilisés les genres envisagés et il exploite surtout des sources rhétoriques : Cicéron, Quintilien, Hermogène, Ménandre le rhéteur, Alexandros ou Georges de Trébizonde.

Dans la perspective de procurer une édition commentée, accompagnée d'une traduction française, des livres I et de la fin du chant III (95-126), co-dirigée avec Michel Magnien (université Paris III), nous avons reçu Luc Deitz (conservateur à la Bibliothèque nationale du Luxembourg et professeur de latin médiéval et de la Renaissance à l'université de Trèves) qui a préparé l'édition allemande des Poetices libri septem, en collaboration avec Gregor Vogt-Spira ${ }^{5}$, pour déterminer des principes d'édition et discuter des difficultés spécifiques à Scaliger. Enfin, Michel Magnien a présenté sa traduction des chapitres I, 56 et 57 et III, 125-126 afin d'analyser les modalités d'analyse spécifiques à chacun des livres.

\section{Georg Sabinus Schüler, dit Sabinus, poète de Charles Quint}

Le deuxième volet du séminaire a été consacré aux premiers poèmes publiés par Georg Schüler (1508-1560), surnommé Sabinus en référence à l'ami d'Ovide dont il fournit un commentaire des Métamorphoses (Fabularum Ovidii interpretatio tradita in Academia regio montana a Georgio Sabino, Vitebergae, ex. offic. haeredum Georgii Rhann, 1555, réédité à Wittenberg, chez C. Schleich et A. Schöne, en 1572,

5. Sieben Bücher über die Dichkunst, éd. L. Deitz et G. Vogt-Spira, Stuttgart, Bad Cannstatt, F. Frommann, 1995-2003. 
et à Paris, chez J. de Marnef, en 1575). Gendre de Philippe Mélanchthon, il fut l'un des poètes les plus féconds du cercle de Wittenberg avant de devenir professeur d'éloquence à Francfort-sur-l'Oder, puis premier recteur de l'université protestante de Königsberg, en $1544^{6}$.

En 1530, paraît à Wittenberg un petit recueil qui se compose d'une dédicace de Joachim Camerarius à Sabinus, composée en grec, d'une lettre de Mélanchthon, adressée à Johann Silberborner, un jeune homme qui a appartenu pendant plusieurs années à son école privée, et de quatre élégies de Sabinus : Epistola Philip. Melanch. de conventu augustano. Aliquot elegiae Georgii Sabini, impressum Wittemberge, apud Iosephum Clug, anno XXX. In- $8^{\circ}$, sign. A-C (BNF : cote MZ-4050). Rédigée au moment de la confession d'Augsburg, la lettre s'ouvre par un éloge de Charles Quint et de son chancelier Mercurino Gattinara, mort en juin 1530, dont le rôle est comparé à celui du jurisconsulte Ulpien auprès de l'empereur Alexandre Sévère. Mélanchthon insiste sur la modération des protestants et souhaite que l'empereur, dont il célèbre aussi la modération, apporte des remèdes aux discordes publiques. C'est dans ce contexte d'affrontement religieux qu'il faut situer les quatre élégies de Sabinus qui accompagna Mélanchthon à la diète d'Augsbourg. La première, adressée à Eobanus Hessus, décrit l'entrée de Charles Quint à Augsburg (De Adventu Caroli V. Caesaris, scripta Augustae ad Eobanum Hessum. Georg Sabinus Arnoldo Burenio. S. D.) ${ }^{7}$ et constitue le pendant poétique des chroniques en prose dont la plus étudiée est celle de Jörg Breu qui est illustrée par une série de bois gravés ${ }^{8}$ et que nous avons comparée à une chronique rédigée en français et parue à Anvers en 1530 («L'Entree de la tressacree Maieste imperialle faicte en la ville de Ausbourg le XV de Iuing l'an M. concq cens et XXX », Anvers 1530. BNF Rés. M. 576). La seconde est consacrée à Magnus III von Mecklenburg (1509-1550) qui fut élu évêque de Schwerin en 1516, mais qui ne commença son administration effective qu'en 1532, en raison de l'absence de confirmation pontificale (Ad illustrem principem ac dominum D. magnum ducem Megalburgensem, scripta a Georgio Sabino). Elle est précédée d'une lettre en prose adressée à Arnold Burenius (1485-1566), un proche de Mélanchthon qui

6. Voir notamment H. Scheible, « Georg Sabinus (1508-1560): ein Poet als Gründungsrektor », dans Die Albertus-Universität zu Königsberg und ihre Professoren, Berlin, 1995, p. 17-31, réimp. dans Melanchthon und die Reformation, Mayence, von Zabern, 1996, p. [533]-547.

7. Sur ce poème, voir W. Kühlmann, « Der Poet und das Reich - Politische, kontextuelle und ästhetische Dimensionen der humanistischen Türkenlyrik in Deutschland », dans Vom Humanismus zur Spätaufklärung. Ästhetische und kulturgeschichtliche Dimensionen der frühneuzeitlichen Lyrik und Verspublizistik in Deutschland, éd. J. Telle, F. Vollhardt et H. Wiegang, Tübingen, Max Niemeyer Verlag, 2006, p. 104-152 et "Reichspatriotismus und humanistische Dichtung », dans Vom Humanismus zur Spätaufklärung. Ästhetische und kulturgeschichtliche Dimensionen der frühneuzeitlichen Lyrik und Verspublizistik in Deutschland, éd. J. Telle, F. Vollhardt et H. Wiegang, Tübingen, Max Niemeyer Verlag, 2006, p. 84-103.

8. «Die Chronik des Malers Georg Preu des Älteren », dans Chroniken der deutschen Städte vom 14. bis ins 16. Jahrhundert, éd. Friedrich Roth, vol. 29, Leipzig, S. Hirzel Verlag, 1906 et Jörg Breu d. Ä, "Einzug in Augsburg 1530, Holzschnitt in zehn Teilen, in Max Geisberg », dans The German singleleaf woodcut 1500-1550, éd. Walter L. Strauss, New York, 1974, t. I, nºs 357-366. Voir notamment, Pia F. Cuneo, Jörg Breu the Elder and the fashioning of Political identity ca. 1475-1536, Leyde, Boston, Cologne, Brill, 1998, p. 139-177 et A. Morrall, Jörg Breu the Elder. Art, culture and belief in Reformation Augsburg, Farnham, Ashgate, 2001. 
étudia à Wittenberg et qui fut à partir de 1523, le professeur de Magnus III. Sabinus lui recommande la cause des lettres et la sienne. Dans la troisième élégie, la Germanie s'adresse au roi Ferdinand, gouverneur des possessions héréditaires des Habsbourg et roi de Bohême et de Hongrie, pour déplorer les dissidences allemandes et la menace turque (Germania ad regem Ferdinandum). Enfin, la dernière élégie, adressée à Johann Silberborner et à son frère Henri, raconte l'histoire du spectre apparu à un pêcheur de la ville de Spire, considéré par Mélanchthon comme un présage d'affrontements sanglants entre catholiques et protestants (Historia spectri Spirensis ad Joannem Silberbornerum et Henricum Silberbornerum fratres).

Afin de nourrir le commentaire, Vincent Zarini (université Paris-Sorbonne) a proposé une synthèse sur la poésie épidictique latine ${ }^{9}$; nous avons ensuite évoqué un autre recueil de Sabinus, paru à Wittenberg, en 1532, toujours chez Rhau, qui comprend les Caesares germanici, des portraits des empereurs germaniques, de Charlemagne à Ferdinand, imités des Caesares d'Ausone, et un poème consacré à une comète vue à Nuremberg par Erasme Ebner, qui a été interprétée comme l'annonce de la victoire de l'empire sur les Turcs ${ }^{10}$. Enfin, John Nassichuk (université d'Ontario) est venu analyser les poèmes encomiastiques d'Euricius Cordus afin de fournir une introduction au long poème que ce dernier adressa à Charles Quint et aux princes allemands pour qu'ils reconnaissent la vraie religion (Ad Invictissimum Imperatorem Carolum Quintum Caesarem Augustum Aliosque Germaniae Proceres, ut veram tandem Religionem agnoscant, Wittenberg, Joseph Klug, 1525).

9. On pourra consulter sur cette question, V. Zarini, «Histoire, panégyrique et poésie, trois éloges de Rome l'éternelle autour de 400 : Ammien Marcellin, Claudien, Rutilius Namatianus », Ktéma, 24, 1999; "Le Prince au miroir des panégyriques versifiés dans la latinité tardive », dans Le Prince au miroir de la littérature politique de l'Antiquité aux Lumières, éd. F. Lachaud, L. Scordia, Mont-SaintAignan, Publications des universités de Rouen et du Havre, 2007, p. 45-67; «Épique et épidictique dans la poésie latine de l'Antiquité tardive ", dans La lyre et la pourpre. Poésie latine et politique de l'Antiquité tardive à la Renaissance, éd. N. Catellani-Dufrêne et M. Perrin, PUR, 2012, p. 17-32.

10. Caesares germanici descripti a Georgio Sabino, (S. 1. n. d.) [Wittenberg, Rhau], Carmen de Cometa qui conspectus est mens. Augusto an. MDXXXI. ad Erasmum Ebnerum Noribergensem. - La dédicace est datée de 1532. Sur les Caesares germanici et leurs modèles, voir V. Leroux, « Des Césars romains aux Césars germaniques : fortune des Caesares d'Ausone chez Caspar Ursinus Velius, Georgius Sabinus et Jacobus Micyllus », dans La réception d'Ausone, org. E. Wolff, Nanterre, 26-27 octobre 2017, à paraître à Bordeaux, aux éditions Ausonius. 